\title{
Survival Differences by Race/Ethnicity and Treatment for Localized Hepatocellular Carcinoma Within the United States
}

\author{
Robert J. Wong · Douglas A. Corley
}

Received: 3 October 2008/ Accepted: 28 November 2008/Published online: 1 January 2009

(C) The Author(s) 2008. This article is published with open access at Springerlink.com

\begin{abstract}
Racial differences among hepatocellular carcinoma survival have been reported, but the etiology behind these disparities remains unclear. Using multi-variable logistic regression analysis, our restrospective cohort study investigated the demographic disparities in survival among localized hepatocellular carcinoma in the United States. From 1998 to 2001, 2,776 cases of localized hepatocellular carcinoma were identified. Significant racial/ethnic disparities in overall survival and utilization of therapies were identified. Compared with non-Hispanic white males, black females were $56 \%$ less likely to survive 3 years (OR 0.44 ; 95\% CI 0.21-0.93). Treatment-specific models also demonstrated disparities, e.g., compared with non-Hispanic whites, Asians receiving transplantation were $77 \%$ more likely to survive 3 years (OR, 1.77; 95\% CI 1.28-2.44). There are significant racial/ethnic disparities in 3-year survival among patients with localized hepatocellular carcinoma. These differences are partially explained by demographic differences in utilization of therapy and in stage-specific survival for each therapy.
\end{abstract}

Keywords Racial disparities - Cancer epidemiology · Survival differences · Primary liver cancer

\footnotetext{
R. J. Wong ( $\square)$

School of Medicine, University of California, San Francisco, 13501 Campus Drive, Oakland, CA 94605, USA

e-mail: RobertWong123@gmail.com

D. A. Corley

Kaiser Permanente Division of Research, Oakland, CA, USA

D. A. Corley

Department of Medicine, Kaiser Permanente Medical Center, San Francisco, USA
}

\section{Introduction}

Hepatocellular carcinoma ranks among the deadliest cancers worldwide [1-7]. Despite advances in screening and early detection, hepatocellular carcinoma retains a poor prognosis, with overall 1- and 5-year survival rates of 23 and 5\%, respectively $[8,9]$. However, small, localized tumors may be more amenable to curative therapy and substantially improved survival [8-13]. The emergence of more effective screening and surveillance protocols, combined with improvements in curative therapy for early stage cancers, provides the opportunity to identify populations that may benefit most from invasive therapies and investigate modifiable disparities in the application of these treatments.

Recent studies have reported racial/ethnic variations in the rising incidence of hepatocellular carcinoma [14-18]. Some have also suggested that racial/ethnic variations exist in survival outcomes among patients diagnosed with this cancer [10-13, 19-26]. However, these studies were limited by the generalizability of their patient population or did not include detailed data on more advanced therapies commonly used in the United States. One of these studies included only Medicare-recipients who, because of age and comorbid conditions, may be less likely to receive aggressive interventions (e.g., resection or liver transplantation). No study to date has focused specifically on localized cancers, the type most likely to respond to therapeutic interventions [10-12, 25, 26]. A detailed analysis of survival after a localized tumor diagnosis would provide the greatest information on whether survival differences exist by race, ethnicity, and gender and whether these differences are due to differences in the use of specific treatments for the same stage of disease, different responses to treatment, or other factors. In the last decade, the 
National Cancer Institute's Surveillance, Epidemiology, and End Results (SEER) cancer registry has incorporated more detailed information on therapeutic interventions; these data now permit a population-based assessment for treatment disparities, treatment responses, and survival for different demographic groups.

We performed a study utilizing high-quality data from the SEER cancer registry to evaluate whether race and ethnicity were associated with survival after the diagnosis of localized stage hepatocellular carcinoma, adjusted for sex, age, year of diagnosis, and treatment type. We then evaluated whether survival differences were explained by geographic or demographic disparities in treatment administered or demographic differences in the response to treatment.

\section{Methods}

\section{Data Sources}

We analyzed data from the SEER registry, a populationbased cancer registry covering approximately $26 \%$ of the US population, for the years 1998-2004 (the most recent year of data). The SEER population is comparable to the general US population with regards to measures of poverty and education [27]. Prior to 1998, the SEER program compiled only basic information for cancer-directed surgical therapies. Starting in 1998, SEER registries added detailed therapeutic interventions such as ablation, transplantation, etc. The 1998-2004 data set includes data from registries in 17 geographic regions: Atlanta, Connecticut, Detroit, Hawaii, Iowa, New Mexico, California (San Francisco-Oakland, Los Angeles, San Jose-Monterey, and Greater California, which includes Central California, Sacramento, Tri-County, Desert Sierra, Northern California, San Diego/Imperial County, Orange County), SeattlePuget Sound, Utah, Rural Georgia, the Alaska Native Tumor Registry, Kentucky, Louisiana, and New Jersey $[27,28]$.

\section{Case Definitions}

Cases of hepatocellular carcinoma were identified using anatomic site (liver: C22.0) and histology codes (hepatocellular carcinoma: 8170-8175) from the International Classification of Disease for Oncology, 3rd ed. [29]. Hepatocellular carcinoma, NOS (8170), accounted for 98.9\% of our cases. Localized cancers were classified using SEER staging criteria [30]; a "localized" SEER stage included cancers confined to one lobe of the liver (with or without vascular invasion), and without evidence of nodal or extrahepatic involvement.

\section{Race/Ethnicity Definitions}

Our analyses utilized the following SEER race and ethnicity categories: non-Hispanic whites, blacks, Asian/ Pacific Islanders (Asian/PI), and Hispanic whites (Hispanics). The small number of cancer cases among other groups (American Indian/Alaskan, black Hispanics, Asian/ PI Hispanics) precluded the calculation of precise estimates for these populations.

\section{Treatment Definitions}

The SEER database includes information regarding the type of therapy received by each patient. Among patients receiving multiple treatments (e.g., radiation prior to resection), only the first treatment is recorded. We grouped therapeutic interventions into five categories: no invasive therapy, local tumor destruction (including photodynamic therapy, electrocautery, cryosurgery, laser, percutaneous ethanol injection, and local tumor destruction not otherwise specified [NOS]), radio frequency ablation, resection (wedge, segmental, or lobectomy), and liver transplantation [30].

\section{Outcome Definitions}

The main outcome was the proportion of persons surviving 3 years after a localized hepatocellular carcinoma diagnosis. This outcome (rather than shorter intervals) was chosen given the overall 3 -year survival for localized hepatocellular carcinoma in our data set was $<30 \%$ and the 3 -year interval decreased the impact of lead time bias from detection for any demographic group. Detailed treatment data and follow-up was available for the years 1998-2004; thus, a case diagnosed in 2001 had 3 years of follow-up available. Longer intervals (e.g., 5 years) provided relatively few cases for analysis.

\section{Statistical Analysis}

Analyses were performed using the SEER*Stat 6.5.3 (National Cancer Institute, Maryland) and Stata statistical packages (release ten, Stata Corporation, Texas). Odds ratios (OR) and 95\% confidence intervals (CI) were calculated using logistic regression models, with an outcome variable of 3-year survival; we did not use hazard ratios to decrease lead or length time biases incorporated into timeto-event analyses. Multivariable logistic regression models were adjusted for sex, age, year of diagnosis, race/ethnicity, and therapeutic intervention. Geography (via SEER registry location) was also evaluated as a potential confounder. Our study focused on the five geographic regions with $>450$ total hepatocellular carcinoma cases (San 
Francisco-Oakland, Detroit, Los Angeles, Greater California, and New Jersey) to ensure enough statistical power to assess differences among treatment distribution between ethnic subgroups.

\section{Results}

\section{Overview}

The mean proportion of persons surviving three years after a localized hepatocellular carcinoma diagnosis during the period 1973-1997 was $18.1 \%$ (95\% CI 15.9-20.3), with the lowest proportion among blacks $(10.0 \%$; 95\% CI 6.5-13.5) and the highest among Asian/PI (23.0\%; 95\% CI 19.926.1). For the 1998-2001 time interval, the 3-year survival proportions for cases diagnosed were higher for the group as a whole (28.1\%; 95\% CI 26.3-29.9) and for each race/ ethnicity group compared with cancers diagnosed between 1973 and 1997 (Table 1).

\section{Distribution of Therapy by Type and Geographic Region}

We investigated whether differences in survival by race/ ethnicity were explained by variations in therapy received. The administration of any invasive intervention was highest among Asian/PI (38.9\%; 95\% CI 35.0-42.1) and lowest among Hispanics (25.5\%; 95\% CI 21.7-29.3) (Table 2). For specific treatments, Asian/PI were the most likely to receive hepatic resection $(24.5 \%$; 95\% CI 21.6-27.9) and Hispanics were the least likely $(9.4 \%$; 95\% CI 7.1-12.3). Non-Hispanic whites were the most likely to receive liver transplantation $(9.4 \%$; 95\% CI 8.0-11.0) and blacks were the least likely $(4.8 \%$; $95 \%$ CI 2.7-7.9). The proportions receiving local tumor destruction therapy and radio frequency ablation were similar among all racial/ethnic groups.

We evaluated whether the differences in treatment received were due to geographic differences. If a given region, for example, was more likely to provide hepatic resection as a treatment and that region had a higher
Table 2 Distribution of invasive therapies administered, stratified by race/ethnicity, among patients with localized hepatocellular carcinoma (years 1998-2004)

\begin{tabular}{|c|c|c|c|}
\hline Therapy & Count & Percent $(\%)$ & $95 \% \mathrm{CI}$ \\
\hline \multicolumn{4}{|l|}{ Non-Hispanic white } \\
\hline No invasive therapy & 1,033 & 67.8 & $65.4-70.2$ \\
\hline Radio frequency ablation & 28 & 1.8 & $1.2-2.6$ \\
\hline Local tumor destruction & 73 & 4.8 & $3.8-6.0$ \\
\hline Resection & 246 & 16.1 & $14.3-18.1$ \\
\hline Transplant & 143 & 9.4 & $8.0-11.0$ \\
\hline \multicolumn{4}{|l|}{ Black } \\
\hline No invasive therapy & 228 & 73.6 & $68.3-78.4$ \\
\hline Radio frequency ablation & 6 & 1.9 & $0.7-4.2$ \\
\hline Local tumor destruction & 15 & 4.8 & $2.7-7.9$ \\
\hline Resection & 46 & 14.8 & $11.1-19.3$ \\
\hline Transplant & 15 & 4.8 & $2.7-7.9$ \\
\hline \multicolumn{4}{|l|}{ Asian/PI } \\
\hline No invasive therapy & 457 & 61.1 & $57.9-65.0$ \\
\hline Radio frequency ablation & 12 & 1.6 & $0.8-2.8$ \\
\hline Local tumor destruction & 47 & 6.3 & $4.7-8.3$ \\
\hline Resection & 183 & 24.5 & $21.6-27.9$ \\
\hline Transplant & 44 & 5.9 & $4.3-7.9$ \\
\hline \multicolumn{4}{|l|}{ Hispanic white } \\
\hline No invasive therapy & 395 & 74.5 & $70.7-78.3$ \\
\hline Radio frequency ablation & 12 & 2.3 & $1.2-3.9$ \\
\hline Local tumor destruction & 29 & 5.5 & $3.7-7.8$ \\
\hline Resection & 50 & 9.4 & $7.1-12.3$ \\
\hline Transplant & 43 & 8.1 & $5.9-10.8$ \\
\hline
\end{tabular}

proportion of Asian/PI, geography may confound the association between hepatic resection and Asians/PI. Geography, however, did not explain the treatment differences seen. The use of specific therapies differed markedly by geographic region between the five SEER regions that reported at least 450 cases, but the general patterns of treatment differences by race/ethnicity were similar within each region (Table 3). Similar to the pooled data from all registries, within each region Asian/PI were generally more likely to receive resection, non-Hispanic whites and Hispanics were more likely to receive transplantation, and

Table 1 Three-year survival in patients diagnosed with localized hepatocellular carcinoma, stratified by race/ethnicity

\begin{tabular}{|c|c|c|c|c|c|c|}
\hline \multicolumn{4}{|c|}{ Cases diagnosed from 1973 to 1997} & \multicolumn{3}{|c|}{ Cases diagnosed from 1998 to 2001} \\
\hline Male and female & 3 year survival $(\%)$ & $95 \% \mathrm{CI}$ & Total cases & 3 year survival $(\%)$ & $95 \% \mathrm{CI}$ & Total cases \\
\hline Non-Hispanic white & 18.1 & $15.9-20.3 \%$ & 1,556 & 28.3 & $25.8-30.8 \%$ & 1,328 \\
\hline Black & 10.0 & $6.5-13.5 \%$ & 324 & 18.8 & $13.9-23.7 \%$ & 275 \\
\hline Asian/PI & 23.0 & $19.9-26.1 \%$ & 770 & 32.6 & $28.9-36.3 \%$ & 697 \\
\hline Hispanic white & 15.4 & $11.5-19.3 \%$ & 364 & 26.1 & $22.0-30.2 \%$ & 476 \\
\hline
\end{tabular}

SEER dataset time periods, 1973-1997 versus 1998-2001 (with subsequent 3-year survival data through 2004) 
blacks were less likely to receive any treatment. For example, in Detroit only $5.2 \%$ of blacks received transplantation compared with $12.8 \%$ of non-Hispanic whites $(P<0.02)$. Even among Asian/PI, who generally were more likely to receive some treatment, significant differences in transplantation were identified (6.2\% in Asians/PI vs. $12.5 \%$ in non-Hispanic whites; Los Angeles, $P<0.01$ ) (Table 3). Age and gender adjusted analyses for the comparisons presented above were all statistically significant $(P<0.05)$.

\section{Adjusted Survival Analyses}

Race/ethnic differences in the proportion surviving 3 years still persisted after adjustment for therapy type, age, and year of diagnosis. For example, compared to non-Hispanic white males (the largest group), black females were $56 \%$ less likely to survive at least 3 years (OR 0.44; 95\% CI 0.21-0.93) and Asian/PI males were 31\% more likely to survive at least 3 years (OR 1.31; 95\% CI 1.00-1.72) (Table 4).
Treatment Response

We evaluated whether the survival disparities were explained by demographic differences in the response to each treatment modality, given adjustment for treatment type alone did not eliminate the survival differences for localized disease. We compared survival for each treatment regimen vs. no treatment, stratified by sex or race/ethnicity, and adjusted for age and year of diagnosis.

The response to local tumor destruction was lower in females than in males (females: 19.5\% 3-year survival, OR 2.00, 95\% CI 0.91-4.39; males: 46.3\% survival, OR 7.57, 95\% CI 5.06-11.33). In contrast, 3-year survival rates were comparable between males and females for surgical resection, transplantation, and radio frequency ablation. For both males and females, patients receiving transplantation were the most likely to survive 3 years (males: $80.2 \%$ survival, OR 26.94, 95\% CI 18.07-40.18; females: $80.4 \%$ survival, OR 19.76, 95\% CI 10.04-38.90) (Table 5).

The response to therapy for local stage disease varied by race/ethnicity, even after adjustment for sex, age, and year

Table 3 Distribution of therapy for SEER registries with $>450$ cases of hepatocellular carcinoma, stratified by race/ethnicity (years 1998-2004)

\begin{tabular}{|c|c|c|c|c|c|c|c|c|c|c|}
\hline \multirow[t]{2}{*}{ SEER Registry } & \multicolumn{2}{|c|}{ No invasive therapy } & \multicolumn{2}{|c|}{ Local destruction } & \multicolumn{2}{|l|}{ RFA } & \multicolumn{2}{|c|}{ Resection } & \multicolumn{2}{|c|}{ Transplant } \\
\hline & Percent & Count & Percent & Count & Percent & Count & Percent & Count & Percent & Count \\
\hline \multicolumn{11}{|l|}{ SF-Oakland } \\
\hline Non-Hispanic white & 67.2 & 178 & 3 & 8 & 3.8 & 10 & 12.8 & 34 & 13.2 & 35 \\
\hline Black & 73.2 & 41 & 0 & 0 & 1.8 & 1 & 14.3 & 8 & 10.7 & 6 \\
\hline Asian & 58.5 & 172 & 1.4 & 4 & 6.1 & 18 & 23.5 & 69 & 10.5 & 31 \\
\hline Hispanic white & 67.1 & 51 & 0 & 0 & 7.9 & 6 & 14.5 & 11 & 10.5 & 8 \\
\hline \multicolumn{11}{|l|}{ Detroit } \\
\hline Non-Hispanic white & 62.4 & 181 & 5.9 & 17 & 3.1 & 9 & 15.9 & 46 & 12.8 & 37 \\
\hline Black & 69.7 & 108 & 7.1 & 11 & 5.2 & 8 & 12.9 & 20 & 5.2 & 8 \\
\hline Asian & 70.6 & 12 & 0 & 0 & 0 & 0 & 23.5 & 4 & 5.9 & 1 \\
\hline Hispanic white & 66.7 & 6 & 0 & 0 & 11.1 & 1 & 11.1 & 1 & 11.1 & 1 \\
\hline \multicolumn{11}{|l|}{ Los Angeles } \\
\hline Non-Hispanic white & 64.8 & 234 & 3.6 & 13 & 3.3 & 12 & 15.8 & 57 & 12.5 & 45 \\
\hline Black & 79.1 & 83 & 1.9 & 2 & 0 & 0 & 10.4 & 12 & 6.7 & 7 \\
\hline Asian & 63.1 & 243 & 5.7 & 22 & 5.5 & 21 & 19.1 & 74 & 6.2 & 24 \\
\hline Hispanic white & 74.1 & 280 & 4.2 & 16 & 1.9 & 7 & 8.7 & 33 & 11.1 & 42 \\
\hline \multicolumn{11}{|l|}{ Greater $C A$} \\
\hline Non-Hispanic white & 64.6 & 506 & 5.5 & 43 & 8.1 & 63 & 12.4 & 97 & 9.3 & 73 \\
\hline Black & 68.6 & 59 & 2.3 & 2 & 7 & 6 & 18.6 & 16 & 3.5 & 3 \\
\hline Asian & 60.9 & 201 & 6.1 & 20 & 9.7 & 32 & 17 & 56 & 5.5 & 18 \\
\hline Hispanic white & 72.4 & 318 & 4.8 & 21 & 5.7 & 25 & 6.4 & 28 & 10.5 & 46 \\
\hline \multicolumn{11}{|l|}{ New Jersey } \\
\hline Non-Hispanic white & 50.3 & 189 & 8.8 & 33 & 9.3 & 35 & 14.1 & 53 & 16.5 & 62 \\
\hline Black & 49.5 & 51 & 19.4 & 20 & 4.9 & 5 & 17.5 & 18 & 8.7 & 9 \\
\hline Asian & 40 & 26 & 7.7 & 5 & 7.7 & 5 & 33.9 & 22 & 9.2 & 6 \\
\hline Hispanic white & 48.1 & 37 & 20.8 & 16 & 6.5 & 5 & 11.7 & 9 & 11.7 & 9 \\
\hline
\end{tabular}


Table 4 Multivariable logistic regression analysis of 3-year survival by sex and race/ethnicity

\begin{tabular}{llllllc}
\hline Predictor variable & 3-year survival \% & Cases & $\begin{array}{c}\text { OR } \\
\text { Age and date adjusted }\end{array}$ & $\begin{array}{c}\text { 95\% CI } \\
\text { Age, date, and treatment adjusted }\end{array}$ \\
\hline Non-Hispanic white male & 27.8 & 998 & 1.00 & Reference & 1.00 & Reference \\
Non-Hispanic white female & 29.7 & 330 & 1.48 & $1.12-1.94$ & 1.19 & $0.86-1.65$ \\
Black male & 21.7 & 193 & 0.65 & $0.45-0.95$ & 0.81 & $0.52-1.24$ \\
Black female & 12.0 & 82 & 0.40 & $0.20-0.80$ & 0.44 & $0.21-0.93$ \\
Asian/PI male & 33.2 & 497 & 1.39 & $1.10-1.76$ & 1.31 & $1.00-1.72$ \\
Asian/PI female & 31.2 & 200 & 1.47 & $1.06-2.05$ & 1.41 & $0.95-2.08$ \\
Hispanic white male & 24.8 & 338 & 0.81 & $0.61-1.08$ & 1.04 & $0.74-1.47$ \\
Hispanic white female & 29.3 & 138 & 1.19 & $0.79-1.79$ & 1.37 & $0.85-2.20$
\end{tabular}

Cases include localized hepatocellular carcinoma diagnosed from 1998 to 2001 (with 3-year follow-up through 2004). The 3-year survival is a crude (unadjusted) value

Table 5 Multivariable logistic regression analysis of 3-year survival by category of invasive therapy received

\begin{tabular}{lllc}
\hline Predictor variable & $\begin{array}{l}\text { 3-year } \\
\text { survival }\end{array}$ & $\begin{array}{l}\text { Adjusted } \\
\text { OR }\end{array}$ & $95 \%$ CI \\
\hline $\begin{array}{l}\text { Male } \\
\text { No invasive therapy }\end{array}$ & $11.9 \%$ & 1.00 & Reference \\
$\begin{array}{l}\text { Radio frequency } \\
\quad \text { ablation }\end{array}$ & $44.1 \%$ & 6.11 & $3.15-11.87$ \\
Local tumor destruction & $46.3 \%$ & 7.57 & $5.06-11.33$ \\
Resection & $53.4 \%$ & 8.98 & $6.86-11.76$ \\
Transplant & $80.2 \%$ & 26.94 & $18.07-$ \\
& & & 40.18 \\
Female & $13.0 \%$ & 1.00 & Reference \\
No invasive therapy & $44.0 \%$ & 2.89 & $0.95-8.84$ \\
Radio frequency & & & \\
$\quad$ ablation & $19.5 \%$ & 2.00 & $0.91-4.39$ \\
Local tumor destruction \\
Resection & $56.8 \%$ & 8.31 & $5.52-12.50$ \\
Transplant & $80.4 \%$ & 19.76 & $10.04-$ \\
& & & 38.90 \\
\hline
\end{tabular}

The 3-year survival proportion is a crude (unadjusted) value. This odds ratio is adjusted for age, year of diagnosis, and race/ethnicity among localized HCC diagnosed from 1998 to 2001 (with 3-year follow-up through 2004)

of diagnosis (Table 6). For any given treatment, compared with non-Hispanic whites, Asian/PI had trends for the greatest overall survival after treatment, whereas blacks had the least benefit, although some confidence intervals overlapped 1.00. For example, Asian/PI who received transplants for limited stage disease were $77 \%$ more likely to be alive 3 -years postdiagnosis than similar non-Hispanic whites matched for age, sex, and year of diagnosis (OR 1.77, 95\% CI 1.28-2.44). In contrast, there was a strong trend for blacks to be less likely to survive 3 years after resection, compared with non-Hispanic whites (OR 0.65, 95\% CI 0.42-1.01). These general trends were similar for all treatment modalities including local tumor destruction
Table 6 Multivariable logistic regression analyzing the association between 3-year survival and category of invasive therapy received stratified by race/ethnicity

\begin{tabular}{lll}
\hline Predictor variable & Adjusted OR & $95 \%$ CI \\
\hline Radio frequency ablation & & \\
Non-Hispanic white & 1.00 & Reference \\
Black & 0.71 & $0.41-1.24$ \\
Asian/PI & 2.03 & $1.45-2.83$ \\
Hispanic white & 1.14 & $0.77-1.69$ \\
Local tumor destruction & & \\
Non-Hispanic white & 1.00 & Reference \\
Black & 0.66 & $0.39-1.13$ \\
Asian/PI & 1.69 & $1.22-2.32$ \\
Hispanic white & 1.22 & $0.85-1.76$ \\
Resection & & \\
Non-Hispanic white & 1.00 & Reference \\
Black & 0.65 & $0.42-1.01$ \\
Asian/PI & 1.50 & $1.16-1.95$ \\
Hispanic white & 1.13 & $0.80-1.58$ \\
Transplant & & \\
Non-Hispanic white & 1.00 & Reference \\
Black & 0.84 & $0.51-1.40$ \\
Asian/PI & 1.77 & $1.28-2.44$ \\
Hispanic white & 1.24 & $0.86-1.78$ \\
\hline The & .517 &
\end{tabular}

The 3-year survival proportion is a crude (unadjusted) value. The odds ratio is adjusted for sex, age, and year of diagnosis among localized HCC diagnosed from 1998 to 2001 (with 3-year follow-up through 2004)

therapy, radio frequency ablation, resection, and transplantation.

\section{Discussion}

Among patients with localized hepatocellular carcinoma, our study identified substantial and significant differences 
by race/ethnicity in overall 3-year survival, type of therapy administered for limited stage disease, and survival after specific therapies. Compared with non-Hispanic whites, blacks were less likely to survive 3 years after diagnosis, less likely to receive any treatment, and, when treated, less likely to survive 3 years for most specific treatment types; the lowest survival times were found in black females. In contrast, Asian/PI had the highest 3-year survival outcomes, were the most likely to receive any treatment (particularly resection), and, when treated, had better 3year survival outcomes for each specific treatment type. Although the likelihood of treatment varied somewhat by geographic region, demographic disparities in receipt of therapy existed in most geographic regions and geography did not explain the overall patterns of survival or treatment by race/ethnicity (Table 3). While our study focused on regions where the number of localized hepatocellular carcinoma cases exceeded 450 for purposes of statistical analysis, the same discrepancies persisted even among more rural areas of the country that were not included in Table 3. For example, in the Kentucky registry, $12.8 \%$ of non-Hispanic whites received liver transplantation compared with $6.3 \%$ of blacks. Similarly, among patients with localized hepatocellular carcinoma in Louisiana, $19.2 \%$ of non-Hispanic whites received transplantation compared with $11.7 \%$ of blacks.

Current estimates of survival outcome among patients diagnosed with hepatocellular carcinoma are concerning. Between 1977 and 1996, 1-year survival improved from 14 to $23 \%$, but overall and longer-term survival remained poor [8]. Patients with localized cancers have the greatest potential for benefit from therapeutic interventions. One study among 4,008 patients diagnosed with hepatocellular carcinoma between 1988 and 1998 reported a 5-year survival of $33 \%$ among those with small, unifocal, nonmetastatic cancers who underwent surgical intervention compared with a 7\% 5-year survival among those who did not receive surgery [11]. The same study noted that $45 \%$ of patients with potentially resectable tumors did not receive surgery. Additional studies investigating treatment outcomes have also suggested possible underutilization of potentially curative therapy among patients with localized cancers [10-13].

The current study extends prior reports that evaluated demographic variables and hepatocellular carcinoma [1026]. A prior study in Medicare patients suggested geographic differences in treatment for hepatocellular carcinoma; however, it is unclear if the results in this population (median age 74 years) can be generalized given older patients may be less likely to receive aggressive interventions such as liver transplantation [10]. Prior studies that utilized the SEER database found racial/ethnic differences in survival overall, but this was prior to the availability of recent detailed data on therapeutic interventions (such as radio frequency ablation, tumor destruction by electrocautery or fulguration, cryosurgery, laser, and percutaneous ethanol injection) that were evaluated in the current study, and there were not detailed analyses of limited stage disease, the stage most responsive to treatment $[10,12,13,26]$. Furthermore, a survival analysis that includes all stages of cancers may be confounded by variations in treatment availability and utility as more aggressive cancers may not be offered invasive therapies. Thus, the current analysis included all ages, expanded data on therapeutic interventions, and focused specifically on localized cancers, which have the greatest potential to demonstrate a survival benefit and to identify any disparities that may exist.

Our findings suggest that demographic differences in the likelihood of receiving treatment and responses to treatment may at least partially underlie the described differences in survival outcome by race/ethnicity. Racial disparities in receipt of surgical therapy have been suggested by others as well [19, 20, 23, 24]. Demographic differences in the delivery of health care are well documented [31, 32]; for example, blacks and women are less likely to receive aggressive cardiac interventions in the presence of coronary artery disease [33-35]. Potential explanations include unequal access to health care, inappropriate overuse in other groups, physician attitudes towards disease risk in different demographic groups, patient attitudes towards medical care, and overt discrimination [36, 37]. In addition, major interventions such as transplantation require the fulfillment of rigid criteria for social support, adherence to medical care, etc.. Meeting these criteria may be more challenging for immigrant groups or persons of lower socioeconomic stature-the groups which are proportionately more likely to include minority populations.

Differences in outcome after specific treatments may also be partially related to the underlying diseases associated with hepatocellular carcinoma, most commonly hepatitis B, hepatitis $\mathrm{C}$, and alcohol abuse. These risk factors are not in the SEER database and could not be evaluated in the current analysis. The biological behavior of hepatocellular carcinoma may differ in the presence of different risk factors. For example, while hepatitis C causing hepatocellular carcinoma is almost always preceded by progressive liver damage resulting in cirrhosis, hepatitis B can lead to hepatocellular carcinoma without liver cirrhosis. These differences likely affect choice of therapy and overall survival. Hepatitis B virus is the main etiological agent for hepatocellular carcinoma in Asians, while hepatitis $\mathrm{C}$ virus is more commonly found in blacks and non-Hispanic whites [38-46]. While few studies have reported variations in surgical interventions, one study suggested that compared to non-Hispanic whites, Asians 
with chronic hepatitis B virus had a significantly higher posttransplantation mortality [23]. A differential response to similar interventions between demographic groups suggests the possibility that ethnicity/race may influence posttherapy survival independent of etiology; such differences are well documented for many disorders, including hypertension and the response to antiviral therapies for hepatitis $C$ [47-50]. Potential explanations for the race/ ethnicity-specific variations in treatment response in our study include differences in disease severity within limited stage disease, host genetic variations to therapy or the cancer, compliance with therapy, disparities in co-interventions that influence survival, or interactions with environmental factors.

Strengths of this study include the utilization of highquality data from a population-based cancer registry that represents a large proportion of the US population [27, 28]. Detailed data on race classifications and therapeutic interventions permitted analysis of sex and race/ethnicityspecific responses to each treatment category and the analyses adjusted for several potential major confounders including age, date of diagnosis, and gender among localized cancers.

There are several potential limitations of this study. In Table 1, we presented improvements in 3-year survival between patients with localized hepatocellular carcinoma diagnosed between 1973 and 1997 and those diagnosed between 1998 and 2001. While the increased survival rates likely represent new advances in cancer targeted therapy and improved techniques of potentially curative interventions, lead time bias may also be another contributing factor. In addition, SEER records the initial therapy received after a cancer diagnosis, but not necessarily the most aggressive therapy. Some patients may have received multiple treatments over time; however, this limitation of all registry studies does not alone explain the race/ethnicity differences in treatment patterns or posttreatment survival. Although all patients analyzed had localized disease, a spectrum of disease exists within each recorded cancer stage. While the SEER category of "localized tumors" is defined by lack of nodal or extrahepatic involvement, it is limited in its ability to identify the extent of tumor involvement (size and unifocal vs. multifocal) within the lobe it affects as well as evidence of vascular invasion. Tumor differences within the category of localized disease may influence the utility of therapy for some patients, although it is not clear that racial/ethnic disparities in the spectrum of disease within each stage would fully account for the differences observed in treatment, treatment response, and overall survival. Even if this were the case, it would suggest there are either biological differences in disease aggressiveness within a specific stage between the different race/ethnic groups or differences in the application of techniques (e.g., screening) between the groups to identify less aggressive cancers. Either of these would be important observations for improving treatment outcomes. Finally, additional factors such as disease etiology (e.g., viral hepatitis), underlying liver disease, severity of liver disease as evidenced by MELD scores, other comorbidities, alcohol use, access to timely care within each SEER region, etc., were not available for analysis; variations in these factors may also influence choices of therapeutic intervention employed or survival and may differ by race/ethnicity.

In summary, for localized stage hepatocellular carcinoma, this study described substantial and significant disparities by race/ethnicity in 3-year survival, therapy administered, and stage-specific survival for individual therapies. The differences were not explained by age, date of diagnosis, or geography. Potential explanations include differences in the delivery of health care between demographic groups with similar stages of disease, disease differences within limited stage disease, disease biology, or variations in treatment response.

Acknowledgments Dr. Wong was supported by funding from the Genentech Foundation for Biomedical Research and the UCSF School of Medicine Dean's Office. The funding sources had no role in the design, conduct, or the collection, management, analysis, and interpretation of the data, or in the preparation, review, or approval of the manuscript for publication.

Open Access This article is distributed under the terms of the Creative Commons Attribution Noncommercial License which permits any noncommercial use, distribution, and reproduction in any medium, provided the original author(s) and source are credited.

\section{References}

1. Parkin DM, Bray F, Ferlay J, et al. Global cancer statistics, 2002. CA Cancer J Clin. 2005;55:74-108.

2. Varela M, Bruix J. Hepatocellular carcinoma in the United States: lessons from a population-based study in Medicare recipients. $J$ Hepatol. 2006;44(1):8-10. doi:10.1016/j.jhep.2005.11.001.

3. Bosch F, Ribes J, Diaz M, et al. Primary liver cancer: worldwide incidence and trends. Gastroenterology. 2004;127 Supp1(5):S5S16. doi:10.1053/j.gastro.2004.09.011.

4. Kiyosawa K, Umemura T, Ichijo T, et al. Hepatocellular carcinoma: recent trends in Japan. Gastroenterology. 2004;127 supp 1(5):S17-S26. doi:10.1053/j.gastro.2004.09.012.

5. Verhoef C, Visser O, de Man RA, et al. Hepatocellular carcinoma in the Netherlands incidence, treatment, and survival patterns. Eur J Cancer. 2004;40(10):1530-1538. doi:10.1016/j.ejca.2004. 03.013.

6. West J, Wood H, Logan RF, et al. Trends in the incidence of primary liver and biliary tract cancers in England and Wales 1971-2001. Br J Cancer. 2006;94(11):1751-1758. doi:10.1038/ sj.bjc. 6603127 .

7. El-Serag HB, Rudolph KL. Hepatocellular carcinoma: epidemiology and molecular carcinogenesis. Gastroenterology. 2007; 132(7):2557-2576. doi:10.1053/j.gastro.2007.04.061. 
8. El-Serag HB, Mason AC, Key C. Trends in survival of patients with hepatocellular carcinoma between 1977 and 1996 in the United States. Hepatology. 2001;33(1):62-66. doi:10.1053/jhep. 2001.21041.

9. Cance WG, Stweart AK, Menck HR. The national cancer database report on treatment patterns for hepatocellular carcinomas. Cancer. 2000;88(4):912-920.

10. El-Serag HB, Siegel AB, Davila JA, et al. Treatment and outcomes of treating of hepatocellular carcinoma among medicare recipients in the United States: a population-based study. $J$ Hepatol. 2006;44(1):158-166. doi:10.1016/j.jhep.2005.10.002.

11. Liu JH, Chen PW, Asch SM, et al. Surgery for hepatocellular carcinoma: does it improve survival? Ann Surg Oncol. 2004;11(3):298-303. doi:10.1245/ASO.2004.03.042.

12. Davila JA, El-Serag HB. Racial differences in survival of hepatocellular carcinoma in the United States: a population-based study. Clin Gastroenterol Hepatol. 2006;4(1):104-110. doi: 10.1016/S1542-3565(05)00745-7.

13. Sloane D, Chen H, Howell C. Racial disparity in primary hepatocellular carcinoma: tumor stage at presentation, surgical treatment and survival. J Natl Med Assoc. 2006;98(12):1934-1939.

14. El-Serag HB, Davila JA, Petersen NJ, et al. The continuing increase in the incidence of hepatocellular carcinoma in the United States: an update. Ann Intern Med. 2003;139(10):817823.

15. Mcglynn KA, Tarone RE, El-Serag HB. A comparison of the trends in the incidence of hepatocellular carcinoma and intrahepatic cholangiocarcinoma in the United States. Can Epid Bio Prev. 2006;15(6):1198-1203. doi:10.1158/1055-9965.EPI-05-0811.

16. Chin PL, Chu DZ, Clarke KG, et al. Ethnic differences in the behavior of hepatocellular carcinoma. Cancer. 1999;85(9):19311936.

17. Di Bisceglie AM, Lyra AC, Schwartz M, et al. Hepatitis Crelated hepatocellular carcinoma in the United States: influence of ethnic status. Am J Gastroenterol. 2003;98(9):2060-2063.

18. Wong R, Corley DA. Racial and ethnic variations in hepatocellular carcinoma incidence within the United States. Am J Med. 2008;121(6):525-531. doi:10.1016/j.amjmed.2008.03.005.

19. Nair S, Eustace J, Thuluvath PJ. Effect of race on outcome of orthotopic liver transplantation: a cohort study. Lancet. 2002;359(9303):287-293. doi:10.1016/S0140-6736(02)07494-9.

20. Harrison LE, Reichman T, Koneru B, et al. Racial discrepancies in the outcome of patients with hepatocellular carcinoma. Arch Surg. 2004;139(9):992-996. doi:10.1001/archsurg.139.9.992.

21. Pignata S, Gallo C, Daniele B, et al. Characteristics at presentation and outcome of hepatocellular carcinoma (HCC) in the elderly: a study of the Cancer of the Liver Italian Program (CLIP). Crit Rev Oncol Hematol. 2006;59(3):243-249. doi: 10.1016/j.critrevonc.2006.01.002.

22. Yuen MF, Yuan HJ, Wong DK, et al. Prognostic determinants for chronic hepatitis B in Asians: therapeutic implications. Gut. 2005;54(11):1610-1614. doi:10.1136/gut.2005.065136.

23. Teo EK, Han SH, Terrault N, et al. Liver transplantation in patients with hepatitis B virus infection: outcome in Asian versus white patients. Hepatology. 2001;34(1):126-132. doi:10.1053/jhep. 2001.25271.

24. Kim WR, Poterucha JJ, Kremers WK, et al. Outcome of liver transplantation for hepatitis B in the United States. Liver Transpl. 2004;10(8):968-974. doi:10.1002/lt.20217.

25. Ananthakrishnan AN, Saeian K. Racial differences in liver transplantation outcomes in the MELD era. Am J Gastroenterol. 2008;103(4):901-910. doi:10.1111/j.1572-0241.2008.01809.x.

26. Siegel AB, McBride RB, El-Serag HB, et al. Racial disparities in the utilization of liver transplantation for hepatocellular carcinoma in the United States, 1998-2002. Am J Gastroenterol. 2008;103(1):120-128.
27. Ries LAG, Melbert D, Krapcho M, eds. SEER Cancer Statistics Review, 1975-2004. Bethesda, MD: National Cancer Institute; 2007.

28. Surveillance, Epidemiology, and End Results (SEER) Program. SEER *Stat database: incidence - SEER 13 regs limited-use, Nov 2006 sub (1992-2004) - linked to county attributes - total U.S., 1969-2004 counties, National Cancer Institute, DCCPS Surveillance Research Program, Cancer Statistics Branch, released April 2007, based on the November 2006 submission. (http://www.seer. cancer.gov).

29. World Health Organization. International Classification of Diseases for Oncology. 3rd ed. Geneva, Switzerland: World Health Organization; 2000.

30. Young JL, Roffers SD, Ries LAG, eds. EER Summary Staging Manual - 2000 Codes and Coding Instructions. Bethesda, MD: National Cancer Institute NIH Pub. No. 01-4969; 2001.

31. Fiscella K, Franks P, Gold MR, et al. Inequality in quality: addressing socioeconomic, racial, and ethnic disparitieis in health care. JAMA. 2000;283(19):2579-2584. doi:10.1001/jama.283. 19.2579.

32. Brown ER, HJKF Foundation. Racial and Ethnic Disparities in Access to Health Insurance and Health care. Los Angeles: UCLA Center for Health Policy Research; 2000.

33. Schneider EC, Leape LL, Weissman JS, et al. Racial differences in cardiac revascularization rates: does "overuse" explain higher rates among white patients? Ann Intern Med. 2001;135(5):328-337.

34. Epstein AM, Weissman JS, Schneider EC, et al. Race and gender disparities in rates of cardiac revascularization: do they reflect appropriate use of procedures or problems in quality of care? Med Care. 2003;41(11):1240-1255. doi:10.1097/01.MLR.000009 3423.38746.8C.

35. Ayanian JZ, Udvarhelyi IS, Gatsonis CA, et al. Racial differences in the use of revascularization procedures after coronary angiography. JAMA. 1993;269(20):2642-2646. doi:10.1001/jama.269. 20.2642.

36. Franks P, Fiscella K. Reducing disparities downstream: prospects and challenges. J Gen Intern Med. 2008;23:672-677. doi:10.1007/ s11606-008-0509-0.

37. Fiscella K, Franks P, Gold MR, Clancy CM. Inequality in quality: addressing socioeconomic, racial, and ethnic disparities in health care. JAMA. 2000;283:2579-2584. doi:10.1001/jama.283.19. 2579.

38. Chen CJ, Yu MW, Liaw YF. Epidemiological characteristics and risk factors of hepatocellular carcinoma. $J$ Gastroenterol Hepatol. 1997;12(9-10):S294-S308. doi:10.1111/j.1440-1746. 1997.tb00513.x.

39. Beasley RP, Hwang LY, Lin CC, et al. Hepatocellular carcinoma and hepatitis B virus a prospective study of 22707 men in Taiwan. Lancet. 1981;2(8256):1129-1133. doi:10.1016/S01406736(81)90585-7.

40. Donato F, Boffetta P, Puoti M. (1998) A meta-analysis of epidemiological studies on the combined effect of hepatitis B and C virus infection in causing hepatocellular carcinoma. Int J Cancer. 1998;75(3):347-354.

41. Villeneuve JP, Desrochers M, Infante-Rivard C, et al. A longterm follow-up study of asymptomatic hepatitis B surface antigen-positive carriers in Montreal. Gastroenterology. 1994;106(4): $1000-1005$.

42. McMahon BJ, Alberts SR, Wainwright RB, et al. Hepatitis Brelated sequelae: prospective study of 1400 hepatitis B surface antigen-positive Alaskan native carriers. Arch Intern Med. 1990;150(5):1051-1054. doi:10.1001/archinte.150.5.1051.

43. Sherman M, Peltekian KM, Lee C. Screening for hepatocellular carcinoma in chronic carriers of hepatitis B virus: incidence and prevalence of hepatocellular carcinoma in a North American urban population. Hepatology. 1995;22(2):432-438. 
44. Alter MJ, Kruszon-Moran D, Nainan OV, et al. The prevalence of hepatitis C virus infection in the United States, 1988 through 1994. N Engl J Med. 1999;341(8):556-562. doi:10.1056/NEJM 199908193410802.

45. Armstrong GL, Simard EP, McQuillan GM, et al. The prevalence of hepatitis C virus infection in the United States, 1999 through 2002. Ann Intern Med. 2006;144(10):705-714.

46. Rustgi VK. The epidemiology of hepatitis $\mathrm{C}$ infection in the United States. J Gastroenterol. 2007;42(7):513-521. doi:10.1007/ s00535-007-2064-6.

47. Park IU, Taylor AL. Race and ethnicity in trials of antihypertensive therapy to prevent cardiovascular outcomes: a systematic review. Ann Fam Med. 2007;5(5):444-452. doi:10.1370/afm.708.
48. The ALLHAT Officers Coordinators for the ALLHAT Collaborative Research Group. Major outcomes in high-risk hypertensive patients randomized to angiotensin-converting enzyme inhibitor or calcium channel blocker vs diuretic: the antihypertensive and lipid-lowering treatment to prevent heart attach trial (ALLHAT). JAMA. 2002;288(23):2981-2997. doi:10.1001/jama.288.23.2981.

49. Mchutchinson J, Poynard T, Pianko S, et al. The impact of interferon plus ribavirin on response to therapy in black patients with chronic hepatitis C. Gastroenterology. 2000;119(5):13171323. doi:10.1053/gast.2000.19289.

50. Fleckenstein J. Chronic hepatitis $\mathrm{C}$ in African Americans and other minority groups. Curr Gastroenterol Rep. 2004;6(1):66-70. doi:10.1007/s11894-004-0028-z. 